Provided for non-commercial research and education use. Not for reproduction, distribution or commercial use.

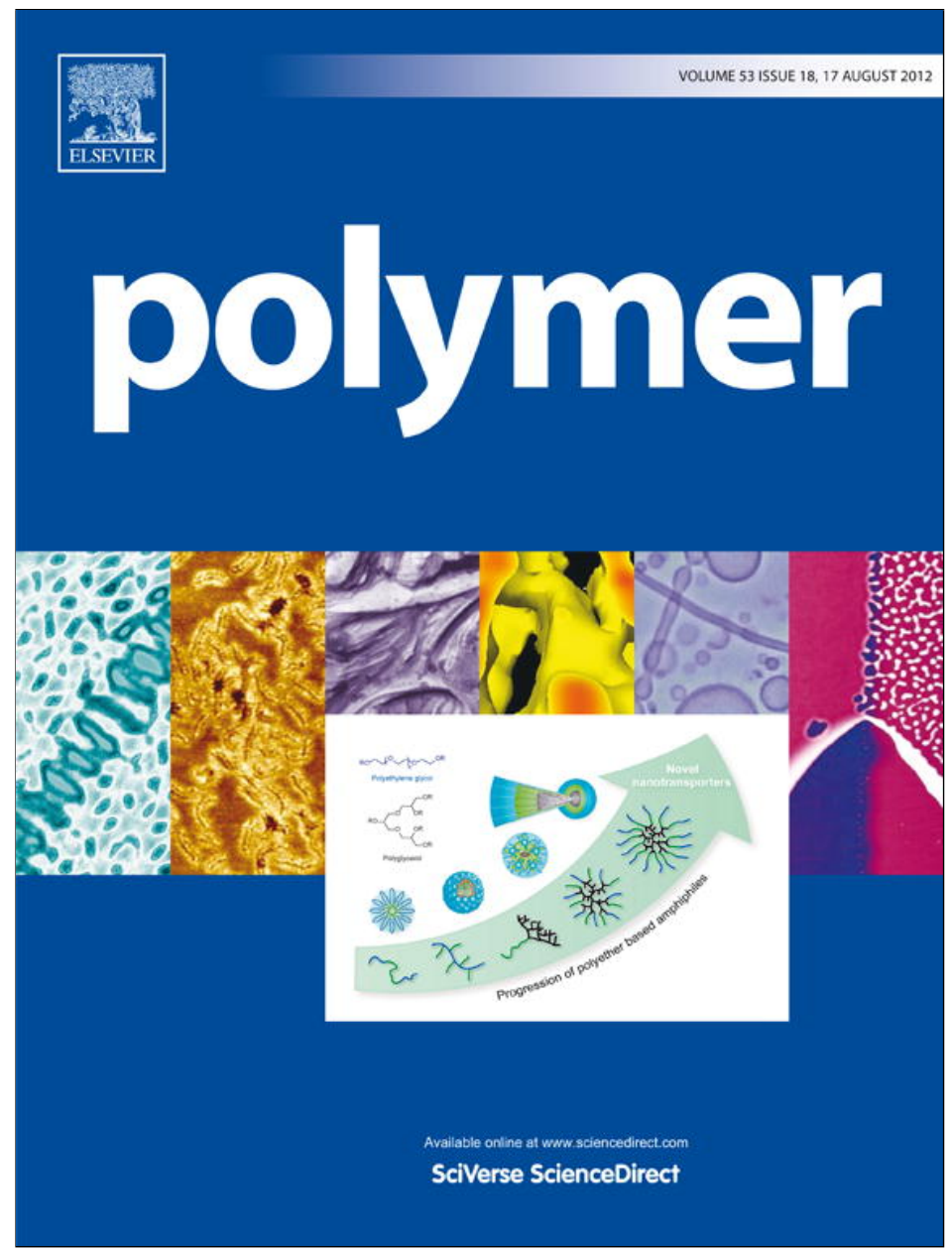

This article appeared in a journal published by Elsevier. The attached copy is furnished to the author for internal non-commercial research and education use, including for instruction at the authors institution and sharing with colleagues.

Other uses, including reproduction and distribution, or selling or licensing copies, or posting to personal, institutional or third party websites are prohibited.

In most cases authors are permitted to post their version of the article (e.g. in Word or Tex form) to their personal website or institutional repository. Authors requiring further information regarding Elsevier's archiving and manuscript policies are encouraged to visit:

http://www.elsevier.com/copyright 


\title{
Confined crystallization of nanolayered poly(ethylene terephthalate) using X-ray diffraction methods
}

\author{
A. Flores $^{\text {a }}$, F. Ania ${ }^{\text {a }}$, C. Arribas $^{\text {b }}$, A. Ochoa ${ }^{\text {c }}$, S. Scholtyssek ${ }^{\text {d }}$, F.J. Baltá-Calleja ${ }^{\text {a,* }}$, E. Baer ${ }^{e}$ \\ ${ }^{a}$ Instituto de Estructura de la Materia, CSIC, Serrano 119, 28006 Madrid, Spain \\ ${ }^{\mathrm{b}}$ Dpto. Materiales y Producción Aeroespacial, E.T.S.I. Aeronáuticos, Universidad Politécnica de Madrid, Pza. Cardenal Cisneros 3, 28040 Madrid, Spain \\ ${ }^{\mathrm{c}}$ Dpto. Química Industrial y Polímeros, E.U.I.T.I., Universidad Politécnica de Madrid, Ronda de Valencia 3, 28012 Madrid, Spain \\ ${ }^{\mathrm{d}}$ Institute of Physics, Martin-Luther-University Halle-Wittenberg, D-06099 Halle (Saale), Germany \\ ${ }^{\mathrm{e}}$ Case Western Reserve University, 2100 Adelbert Road, Cleveland, OH 44106-7202, USA
}

\section{A R T I C L E I N F O}

\section{Article history:}

Received 23 February 2012

Received in revised form

23 May 2012

Accepted 27 June 2012

Available online 4 July 2012

\section{Keywords:}

Nanolayered PET

SAXS

Confinement

\begin{abstract}
A B S T R A C T
The development of crystalline lamellae in ultra-thin layers of poly(ethylene terephthalate) PET confined between polycarbonate (PC) layers in an alternating assembly is investigated as a function of layer thickness by means of X-ray diffraction methods. Isothermal crystallization from the glassy state is insitu followed by means of small-angle X-ray diffraction. It is found that the reduced size of the PET layers influences the lamellar nanostructure and induces a preferential lamellar orientation. Two lamellar populations, flat-on and edge-on, are found to coexist in a wide range of crystallization temperatures $\left(T_{C}=117-150{ }^{\circ} \mathrm{C}\right)$ and within layer thicknesses down to $35 \mathrm{~nm}$. Flat-on lamellae appear at a reduced crystallization rate with respect to bulk PET giving rise to crystals of similar dimensions separated by larger amorphous regions. In addition, a narrower distribution of lamellar orientations develops when the layer thickness is reduced or the crystallization temperature is raised. In case of edgeon lamellae, crystallization conditions also influence the development of lamellar orientation; however, the latter is little affected by the reduced size of the layers. Results suggest that flat-on lamellae arise as a consequence of spatial confinement and edge-on lamellae could be generated due to the interactions with the PC interface.
\end{abstract}

(C) 2012 Elsevier Ltd. All rights reserved.

\section{Introduction}

The current trends in Polymer Science concerning the development of nano-scale materials require an understanding of the physical processes taking place under severe spatial restrictions. The variety of nanostructured polymer systems is diverse; among these, multilayer materials represent one of the simplest models to explore the influence of spatial confinement on the internal structure of the polymer. In turn, this will control the final properties.

Layer-multiplying coextrusion has been successfully employed in the past to produce thousands of highly regular alternating polymer layers with individual thicknesses than can approach only a few nanometres [1]. The method offers a simple route for the combination of properties of immiscible homopolymers. In comparison with thin films, multilayered polymer systems offer the advantage that conventional techniques typically employed for

\footnotetext{
* Corresponding author. Tel.: +34 917459507; fax: +34 915642431.

E-mail addresses: fj.balta@csic.es, embalta@iem.cfmac.csic.es (F.J. Baltá-Calleja).
}

bulk materials can be used. As a further point, interactions with the substrate and the air can be disregarded; instead, polymer interfaces should play a fundamental role.

A general agreement is found in the literature that the morphology and the nanostructure of a polymer material are influenced by finite size effects. Preferential lamellar orientation, slower kinetics of crystallization and lower levels of crystallinity are some of the morphological features commonly appearing with decreasing size of the polymer domains [2,3]. The underlying mechanisms are still a matter of controversy; in the case of thin films, the different substrate-polymer interaction is possibly the reason for the apparent disagreement among several investigations [2].

Preferential lamellar orientation is usually detected in thin (below $1000 \mathrm{~nm}$ ) and ultrathin (below $100 \mathrm{~nm}$ ) polymer layers, either deposited on a substrate or as part of a free-standing multilayer assembly $[2,4,5]$. Edge-on and/or flat-on lamellae (the chain alignment is parallel or perpendicular to the layer plane respectively) arranged in a number of superstructures are usually observed [2]. As a general trend, decreasing the film thickness and increasing the crystallization temperature promote flat-on 
Table 1

PET layer thickness as measured by means of USAXS [10], film thickness, PET/PC ratio and number of layers for the different PET/PC multilayered films.

\begin{tabular}{llll}
\hline $\begin{array}{l}\text { PET thickness } \\
{[\mathrm{nm}]}\end{array}$ & $\begin{array}{l}\text { Film thickness } \\
{[\mu \mathrm{m}]}\end{array}$ & $\begin{array}{l}\text { PET/PC } \\
\text { ratio }\end{array}$ & $\begin{array}{l}N \text { of } \\
\text { layers }\end{array}$ \\
\hline- & 80 & $100 / 0$ & 1024 \\
115 & 90 & $70 / 30$ & 1024 \\
80 & 95 & $50 / 50$ & 1024 \\
50 & 100 & $30 / 70$ & 1024 \\
35 & 170 & $30 / 70$ & 4096 \\
\hline
\end{tabular}

orientation and a number of mechanisms has been suggested $[4,6-8]$. It is clear that as the thickness of the polymer film approaches the dimensions of the lamellar thickness, the appearance of flat-on lamellae should be favoured. According to the diversity of experimental observations, the transition from one orientation to the other seems to depend on the polymer material, the crystallization conditions and, in case of supported films, on the interaction of the material with the substrate [2]. In addition, it has also been reported that both orientations sometimes coexist $[5,9]$.

The aim of the present work is the study of the development of PET lamellae under extreme spatial restrictions and for different crystallization conditions. Coextruded multilayered materials of alternating poly(ethylene terephthalate) (PET) and polycarbonate (PC) with PET layer thicknesses ranging from $\sim 100 \mathrm{~nm}$ down to $\sim 35 \mathrm{~nm}$ are here investigated. The glassy PC material confines the PET layers and preserves the integrity of the multilayered structure up to temperatures around $165{ }^{\circ} \mathrm{C}$ (slightly above the glass transition of PC) [10]. Thus, crystallization of PET from the glassy state can be carried out in a temperature window between the glass transition of PET and that of PC. Preceding studies on a similar PET/PC system suggest that edge-on and flat-on lamellae are simultaneously generated in a $65 \mathrm{~nm}$ layered material for a crystallization temperature of $150{ }^{\circ} \mathrm{C}$ [9]. In the present study we wish to carry out a comprehensive investigation of the development of orientation and lamellar structure of PET as the size of the layer thickness is reduced to only $35 \mathrm{~nm}$ and the crystallization temperatures varied in the range $117^{\circ} \mathrm{C}-150{ }^{\circ} \mathrm{C}$. It is noteworthy that a layer thickness of only $35 \mathrm{~nm}$ is close to the size of a lamellar stack, taking into account that long period values of $\sim 10 \mathrm{~nm}$ and $\sim 3$ crystals per lamellar stack are usually reported for PET [9]. Such small PET layer thicknesses within multilayered systems have not been studied so far. The fact that we can tune the layer thickness while the interfaces remain unchanged seems to be an adequate approach to differentiate the influence of both factors. Small-angle
X-ray diffraction studies to in-situ follow the appearance and evolution of lamellar arrangements are reported. In addition, postcrystallization wide-angle X-ray scattering measurements are also included and discussed.

\section{Experimental}

\subsection{Materials}

Poly(ethylene terephthalate) (PET) (M\&G Cleartuf 8004) and polycarbonate (PC) (DOWCalibre 200-10) were used in this work. Both materials have a molecular weight of $M_{\mathrm{w}} \approx 30,000 \mathrm{~g} / \mathrm{mol}$. The layer multiplying coextrusion process used to prepare the PET/PC nanolayered materials has been described previously [11]. Table 1 collects the characteristics of the different films investigated. All the films exhibited at least two Ultra Small Angle X-ray Scattering (USAXS) maxima accounting for the high regularity of the layered structure [10]. The long spacing associated to the inner maxima directly relates to the repeating distance across the layered structure. The PET layer thicknesses in Table 1 were derived using the corresponding USAXS long spacing and the volume fraction of PET material. For the sake of comparison, a control PET sample, coextruded under the same conditions of the PET/PC sample, was also prepared.

\subsection{Small angle $X$-ray scattering}

\subsubsection{Isothermal crystallization experiments}

As-processed PET and PC within the multilayered structure were amorphous. Real time small-angle X-ray scattering (SAXS) measurements were carried out at the BW4 beamline of DESY, HASYLAB, Hamburg, in order to follow the kinetics of lamellar crystallization at $117{ }^{\circ} \mathrm{C}, 132{ }^{\circ} \mathrm{C}$ and $150{ }^{\circ} \mathrm{C}$. The samples were positioned with the stacking of the layers parallel to the beam direction (MD or TD) in order to properly detect the appearance of lamellar orientation (see Fig. 1). Preceding investigations show that directing the incident beam perpendicularly to the surface of the films (ND) yields isotropic scattering rings $[9,12]$. The wavelength was $0.139 \mathrm{~nm}$ and we used a sample-detector distance of $1640 \mathrm{~mm}$. A MarCCD detector with $2048 \times 2048$ pixels and a pixel size of $79.1 \mu \mathrm{m}$ was employed. A Linkam hot-stage (model THMS600) with a sample holder especially designed to allow the positioning of the films was placed in the sample environment. Samples were rapidly heated at $40{ }^{\circ} \mathrm{C} / \mathrm{min}$ from room temperature up to $T_{c}$.
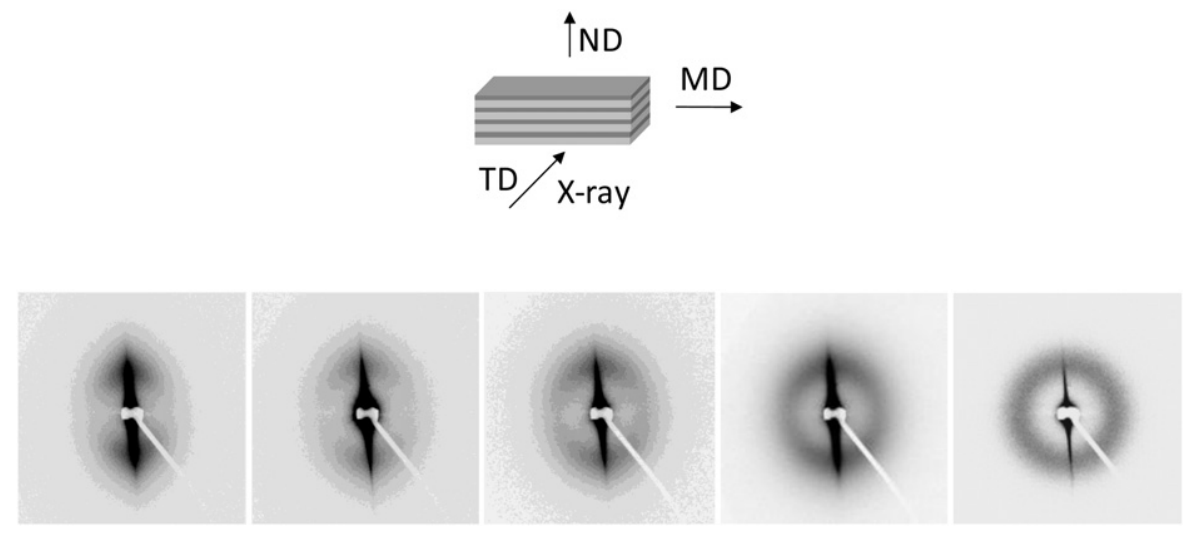

Fig. 1. SAXS patterns of multilayered PET/PC at the end of the crystallization process of PET within individual PET layers of average thickness (from left to right): 35 nm, 50 nm, $80 \mathrm{~nm}, 115 \mathrm{~nm}$ and PET control material. The isothermal crystallization was carried out at $150{ }^{\circ} \mathrm{C}$ for (from left to right): $90 \mathrm{~min}, 90 \mathrm{~min}, 60 \mathrm{~min}, 60 \mathrm{~min}$ and $40 \mathrm{~min}$. The position of the film with respect to the incident beam is illustrated schematically. 


\subsubsection{Lamellar orientation}

Oriented SAXS patterns were obtained at the end of each crystallization process for all the PET/PC multilayered materials investigated. No orientation was found in case of the control PET sample. As an example, Fig. 1 shows the 2D-images corresponding to the last frame of each isothermal crystallization experiment carried out at $150{ }^{\circ} \mathrm{C}$ on the nanolayered series and the control material. The incident beam was directed along TD. Similar diffraction patterns were obtained when the beam was directed along MD. In each case, the crystallization time was maintained for at least $1 \mathrm{~h}$ except for the PET control material where the crystallization kinetics was expected to be the fastest and therefore measurements were interrupted after $\sim 40 \mathrm{~min}$. The strong meridional streak is associated with grazing incidence scattering arising from the interfaces between the two homopolymers, probably due to the presence of nanogaps, and from the outer surfaces. In order not to saturate the detector, the intensity of this meridional streak was reduced by tilting the TD-MD plane of the samples a few degrees away $\left(\sim 5^{\circ}\right)$ from the incident beam.

The FIT2D software package was used to integrate the scattered intensity over a range of wave vectors $q(q=4 \pi \sin \theta / \lambda)$ around the SAXS maximum to yield a plot of intensity as a function of the azimuthal angle $\phi$ (from $0^{\circ}$ to $360^{\circ}$ ) [13]. A least-squares analysis is used to separate the experimental curve into three different contributions: i) a Gaussian function describing the distribution of lamellar orientations, ii) a Lorentzian function associated to the meridional streaks and iii) a constant function accounting for the background (see reference [9] for more details). It should be noted that the criteria adopted to assign the background can significantly affect the distribution of lamellar orientations [14]. For instance, it is common practice to assume the background level as the minimum of the azimuthal intensity profile. However, such procedure can result in a too sharp distribution for low levels of orientation [14], as those displayed by some of our samples. For this reason we adopted the alternative criteria of including the background level as part of the fitting process. The error introduced in this way in the orientation distribution function values (see definition below) has been evaluated and the corresponding error bars are reported. The orientation distribution function was calculated following:

$f=\frac{3<\cos ^{2} \phi>-1}{2}$

The $f$ function provides information on the distribution of orientations along a given preferential direction.

On the other hand, the amount of oriented material was estimated by assuming that the background intensity in the azimuthal scan, once subtracted the background of the first frame (with no apparent SAXS maxima), is associated to the contribution of isotropic crystallized material. The parameter describing the fraction of oriented material, $\chi_{o r}$, is calculated from the ratio between the area under the Gaussian profile and that under the background plus the Gaussian profile.

The kinetics of crystallization was explored by defining a relative crystallinity index, $X_{c}$, as the ratio between the area under the experimental azimuthal scan at the time of interest and that under the azimuthal profile of the last frame. The azimuthal scans were first subtracted for background and scattering associated to the meridional streaks. Note that $X_{c}$ is always 1 at the end of crystallization irrespective of the total amount of crystallized material.

\subsubsection{Lamellar nanostructure}

For the estimation of the average lamellar long period $L$, SAXS patterns were azimuthally integrated with the help of FIT2D software package to obtain a curve of intensity, $I$, as a function of $q$. A plot of $I q^{2}$ versus $q$ was fitted to a Pearson IV function using leastsquares analysis. The position of the maximum of this function was used for the calculation of the long period. Both the azimuthal integration of the intensity and Lorentz correction should be strictly applied only to isotropic systems or weakly oriented diffraction patterns [15] which seem to be the case in most of our experiments. However, larger degrees of orientation were found for samples with the thinnest PET layers as will be discussed below. For the sake of comparison, the procedure described above (azimuthal integration and Lorentz correction) was applied to all the diffraction patterns.

\subsection{Wide angle $X$-ray scattering}

The crystal orientation and crystal structure upon crystallization were investigated by means of wide-angle X-ray scattering (WAXS) measurements. Room temperature two-dimensional patterns of the crystallized materials were obtained using a Micro Star rotating anode generator with copper target manufactured by Bruker (Germany). The wavelength of the beam was $\lambda=0.1542 \mathrm{~nm}$. WAXS patterns were recorded using a Mar345 image plate with a resolution of $3450 \times 3450$ pixels and $100 \mu \mathrm{m} /$ pixel; a sample-to-detector distance of $200 \mathrm{~mm}$ was used.

\section{Results and discussion}

\subsection{Influence of layer thickness on the lamellar orientation}

The SAXS patterns illustrated in Fig. 1 show a clear Bragg maximum associated to the lamellar periodicity developed at the end of each crystallization process at $150{ }^{\circ} \mathrm{C}$. The intensity of this maximum is higher in the meridian except for the PET control material where a uniform distribution along the azimuthal angle is found. Results for the nanolayered materials suggest a preferential orientation of the lamellae with the normal to the basal surfaces along ND (flat-on lamellae). The preferential orientation shows uniaxial symmetry, i.e., flat-on lamellae are randomly oriented in the plane of the layer stacking. The result is in agreement with preceding studies for a $65 \mathrm{~nm}$ nanolayered material [9]. In the present paper, we further show that the same lamellar population is retained for layer thicknesses as thin as $35 \mathrm{~nm}$. Moreover, a quick look to the patterns of Fig. 1 suggests that the arching of the SAXS maxima is reduced as the layer thickness decreases, in other words, severe spatial restrictions enhance lamellar orientation. Indeed, the following SAXS analysis in terms of the orientation distribution function substantiates this contention.

Fig. 2 shows the time evolution of the SAXS orientation distribution function during crystallization at $150{ }^{\circ} \mathrm{C}$ for all the nanolayered materials investigated. The inset of Fig. 2 illustrates the variation of the $f$-parameter, taken at the end of the crystallization process, as a function of the layer thickness, $h$. Error bars illustrate the range of values that $f$ can adopt depending on the background criteria assumed for the analysis of the intensity azimuthal profiles (the background was either taken as the minimum of the intensity azimuthal profile or included as a variable in the fitting process as detailed in the experimental section). The inset clearly shows that the narrower distributions of lamellar orientation are found for the thinner layers. This behaviour is retained upon cooling the sample to room temperature in agreement with preceding ex-situ room temperature SAXS studies in similar multilayer systems [11]. The fact that the order parameter $f$ tends to increase with decreasing layer thickness supports the idea that flat-on lamellae arise as a consequence of spatial confinement and not due to the interactions with the PC interface. The latter case would yield similar 


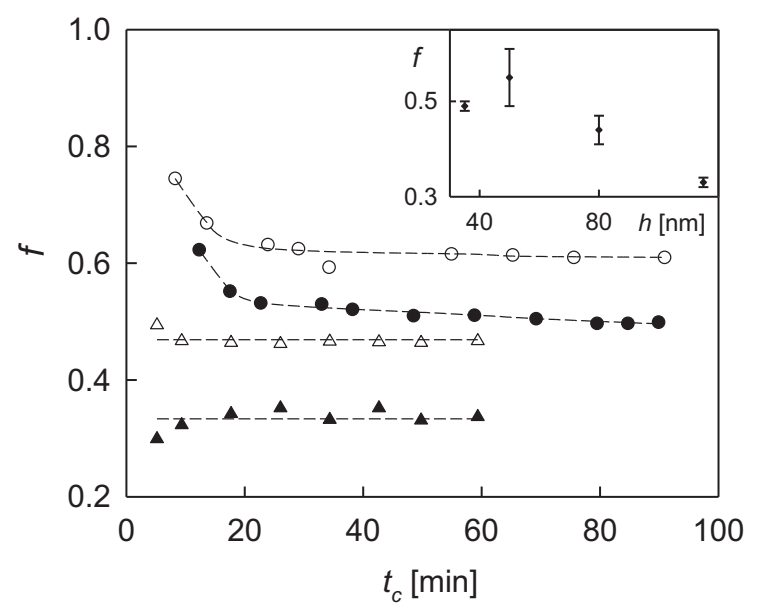

Fig. 2. Orientation distribution function of the PET lamellae along the ND direction versus crystallization time at $150{ }^{\circ} \mathrm{C}$ within the nanolayered materials with PET layer thickness: $(\bullet) 35 \mathrm{~nm},(\bigcirc) 50 \mathrm{~nm},(\Delta) 80 \mathrm{~nm}$ and $(\Delta) 115 \mathrm{~nm}$. Dashed lines are merely eye-guides. The inset shows the $f$-values with their corresponding error bars as a function of PET layer thickness, $h$, at the end of the crystallization process at $150{ }^{\circ} \mathrm{C}$.

values for the orientation function $f$. Fig. 2 also shows that for the thinnest layers, the orientation function initially decreases and finally levels-off after $\sim 20 \mathrm{~min}$, similarly to the behaviour found in case of the $65 \mathrm{~nm}$ layered material [9]. The broadening of maximum in the course of the crystallization process could be attributed to a lamellar insertion mechanism where the inserted lamellae exhibit basal surfaces slightly inclined with respect to the layers surfaces. Fig. 2 shows that such $f$-decrease cannot be clearly observed in case of the thicker PET layers ( 80 and $115 \mathrm{~nm}$ ). In this case, the broader distribution of lamellar orientations ( $f$ values are significantly lower, approaching $f=0.35$ in some cases) indicates a higher degree of randomization. For lamellar stacks with the normal to the lamellar surfaces significantly away from ND, the newly inserted inclined lamellae should not produce such significant decrease in $f$.

Fig. 3 shows the evolution of the fraction of oriented material with crystallization time at $150{ }^{\circ} \mathrm{C}$ for all the PET/PC films investigated. The amount of oriented crystallized material $X_{o r}$ initially increases and subsequently levels-off. It is found that the $X_{o r}$ value is stabilized at a later stage for the thinnest layer thicknesses. This result could be related to the slower kinetics of crystallization

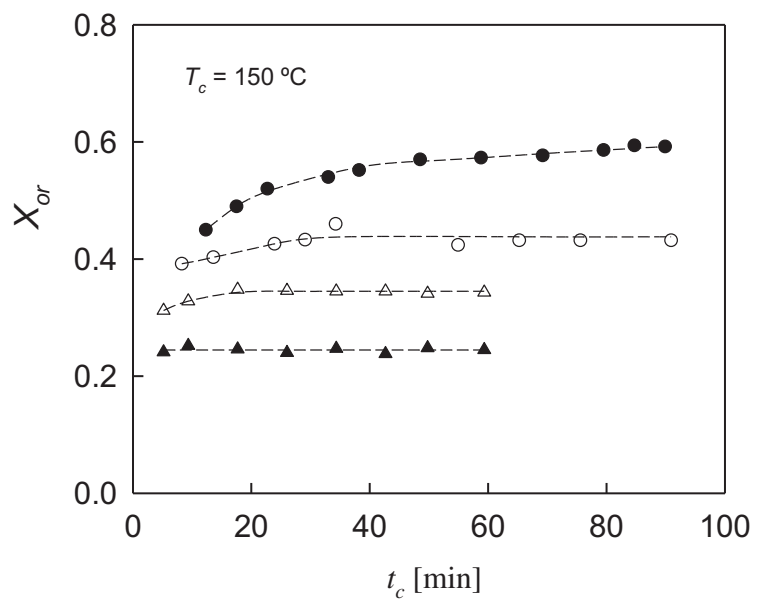

Fig. 3. Fraction of oriented lamellae (relative to the total crystallized lamellae) as a function of crystallization time at $150{ }^{\circ} \mathrm{C}$ for the different multilayered films. Symbols as in Fig. 2. Dashed lines are eye-guides. taking place as layer thickness diminishes, a point that will be discussed below. On the other hand, the fraction of oriented crystallized material is found to be the largest under severe spatial restrictions. Hence, not only the distribution of preferential orientation of the lamellae along the normal to the film but also the amount of oriented material within the crystallized fraction is enhanced as the layer thickness is reduced. In our view, at an early stage during lamellar growth, spatial restrictions would force the lamellae to deflect leading to an overall flat-on orientation. As the layer thickness is reduced the lamellae would bend at an earlier stage, hence, yielding a higher preferential orientation of the lamellae and a larger amount of crystallized oriented material.

\subsection{Influence of crystallization temperature on the development of orientation}

Fig. 4 shows the evolution of the orientation parameter with crystallization time for the multilayered material with $50 \mathrm{~nm}$ PET layer thickness crystallized at $117^{\circ} \mathrm{C}, 132{ }^{\circ} \mathrm{C}$ and $150{ }^{\circ} \mathrm{C}$. Due to the fact that significantly lower levels of crystallinity develop at $117{ }^{\circ} \mathrm{C}$ and $132^{\circ} \mathrm{C}$ with respect to those at $150^{\circ} \mathrm{C}$, the first SAXS frames of the former crystallization experiments exhibit a large noise to signal ratio and hence, have not been included in Fig. 4. Unfortunately, this precludes any precise discussion on the first stages of crystallization and it is difficult to assess whether at low temperatures the orientation function exhibits the same behaviour with time as that found at $150{ }^{\circ} \mathrm{C}$. At large crystallization times, comparison of the $f$ values for each crystallization temperature, $T_{c}$, indicates that the distribution of lamellar orientations broadens with decreasing crystallization temperature. This can be explained taking into account that, as the crystallization temperature diminishes from $150{ }^{\circ} \mathrm{C}$ to $117{ }^{\circ} \mathrm{C}$, the nucleation density is enhanced and the crystal growth rate decreased as shown in preceding cold crystallization studies on bulk PET [16]. At lower crystallization temperatures, spatial restrictions would less effectively orient lamellar crystals (having smaller lateral dimensions) and as a consequence a wider distribution of lamellar orientations is found.

It should be noted that preceding studies in thin and ultrathin polymer films suggest that low $T_{C}$ values favour edge-on lamellae [2]. A transition from flat-on to edge-on lamellae with the decrease of crystallization temperature has even been identified for a $33 \mathrm{~nm}$ poly(bisphenol A hexane ether) film and a nanolayered

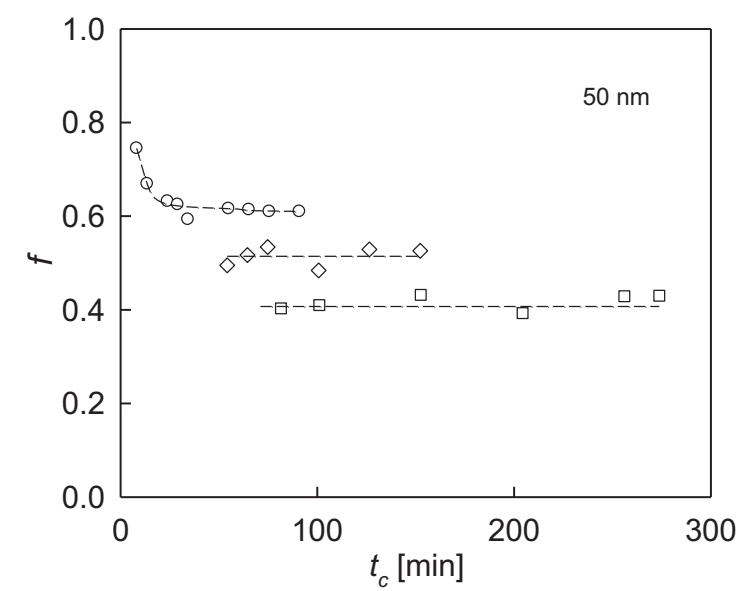

Fig. 4. Plot of the orientation distribution function versus crystallization time for the $50 \mathrm{~nm}$ PET layered sample crystallized at: $(O) 150{ }^{\circ} \mathrm{C},(\diamond) 132{ }^{\circ} \mathrm{C}$ and $(\square) 117{ }^{\circ} \mathrm{C}$. Dashed lines are eye-guides. 
poly(ethylene oxide) (PEO) system [2,17]. Our experimental observations reveal the occurrence of flat-on lamellar crystals within $50 \mathrm{~nm}$ layered PET in a wide range of crystallization temperatures down to $117{ }^{\circ} \mathrm{C}$. Moreover, the development of anisotropic SAXS maxima associated to the occurrence of flat-on lamellae has also been observed for the $115 \mathrm{~nm}$ layered material crystallized at $117^{\circ} \mathrm{C}$ (results not shown here). The latter represents the most unfavourable conditions (thickest layers, lowest $T_{C}$ ) for the development of flat-on lamellae according to the above mentioned studies.

\subsection{Kinetics of crystallization in nanolayered PET}

Fig. 5 (left) shows the increase in relative crystallinity $X_{c}$ with increasing crystallization time at $150{ }^{\circ} \mathrm{C}$ for the different nanolayered materials. It is clearly seen that the kinetics of crystallization is retarded for the thinnest layered samples. Spatial boundary conditions are found to delay the crystallization process. The result could be due to the developing lamellae trying to find new paths for the crystal growth leading to the preferential alignment of the basal planes parallel to the surface layers. This mechanism should be similar to that described in the literature for nano-scaled polymers in which the growing spherulites are truncated at the interfaces [18]. In addition, a decreasing film thickness could reduce the mobility of the polymer chains hindering the incorporation of new stems to the crystal growth front and contributing to the delayed crystallization kinetics observed. Other studies in confined polymers also attribute the delayed crystallization kinetics to changes in the nucleation step $[18,19]$. For instance, a decrease in the number of nuclei has been proposed to occur for films with thicknesses in the range of the critical nuclei size [19]. Moreover, a recent study on confined PEO suggests that the area nucleation density decreases with the layer thickness giving rise to large lamellae; this effect has even been observed for layers in the micron range [18]. The present study does not contemplate the experimental evaluation of the nucleation density effect. However, in contrast to the observations on PEO, our results suggest distorted flat-on lamellae with limited lateral crystal size (see WAXS results below).

Fig. 5 (right) shows the variation of $X_{c}$ with crystallization time for the $50 \mathrm{~nm}$ nanolayered material at different crystallization temperatures. Similarly to bulk PET, the kinetics of crystallization slows down as $T_{C}$ decreases. In addition, the development of crystallinity for nanolayered PET is substantially delayed as compared to bulk PET. Indeed, while preceding studies show that the

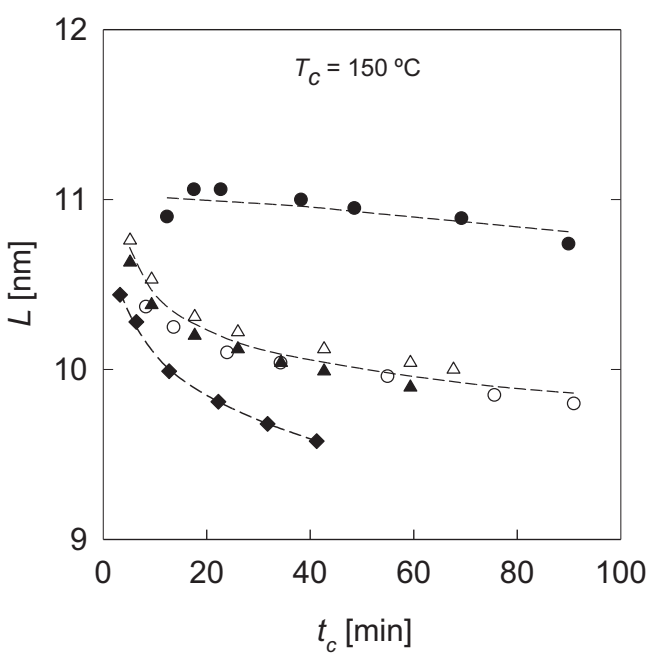

Fig. 6. Variation of average lamellar long periods with crystallization time at $150^{\circ} \mathrm{C}$ for the multilayered materials with PET layer thicknesses of: $(\bullet) 35 \mathrm{~nm},(\bigcirc) 50 \mathrm{~nm}$ $(\Delta) 80 \mathrm{~nm},(\Delta) 115 \mathrm{~nm}$ and $(\diamond)$ PET control material. Dashed lines are eye-guides.

crystallization half time, $\tau_{1 / 2}$, for bulk PET at $127{ }^{\circ} \mathrm{C}$ is of $\tau_{1 / 2} \approx 4 \mathrm{~min}$ [16], the one for the $50 \mathrm{~nm}$ nanolayered material (Fig. 5) is $\tau_{1 / 2} \approx 70 \mathrm{~min}$ for $T_{c}=132{ }^{\circ} \mathrm{C}$ (at $127^{\circ} \mathrm{C}, \tau_{1 / 2}$ would have been even longer). These results confirm the remarkable decrease of crystallization rate found for restricted geometries.

\subsection{Effect of layer thickness on the structure of flat-on lamellae}

Fig. 6 illustrates the variation of the lamellar periodicity $L$ as a function of crystallization time at $150{ }^{\circ} \mathrm{C}$ for all the nanolayered materials. An initial steep $L$ decrease followed by a level-off has been observed in a number of studies concerning the crystallization of bulk isotropic and oriented PET [20-23]. The behaviour has been associated to a diversity of mechanisms: appearance of new lamellar stacks between the existing ones, new single lamellae inserted in the primary stacks, changes in the distribution of crystalline thicknesses or variations in the amorphous layers thicknesses [20-23]. In Fig. 6, most interesting is the observation that the $L$ behaviour with crystallization time for the thinnest nanolayered material is significantly different from the rest of the samples investigated, i.e. $L$ decreasing at a lower rate. It seems that

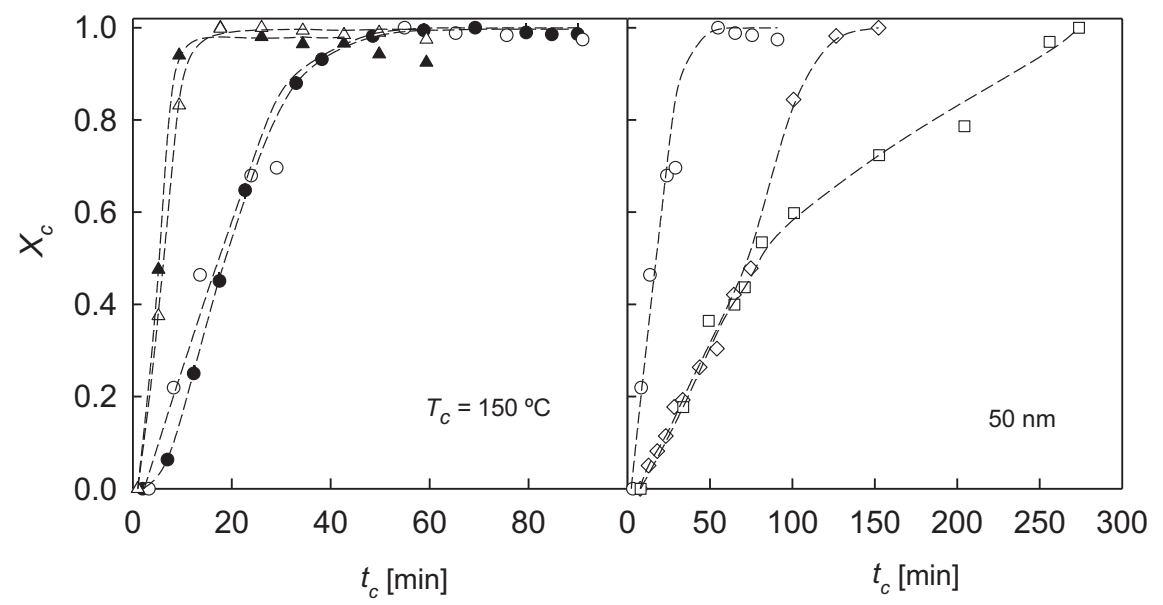

Fig. 5. Relative crystallinity versus crystallization time for: (left) the nanolayered samples with PET layer thickness of $35 \mathrm{~nm}(\bullet), 50 \mathrm{~nm}(O), 80 \mathrm{~nm}(\Delta)$ and $115 \mathrm{~nm}(\boldsymbol{\Delta})$, crystallized at $150{ }^{\circ} \mathrm{C}$; (right) the $50 \mathrm{~nm}$ layered material crystallized at the temperatures of $150{ }^{\circ} \mathrm{C}(O), 132{ }^{\circ} \mathrm{C}(\diamond)$ and $117{ }^{\circ} \mathrm{C}(\square)$. Dashed lines are eye-guides. 
the mechanism underlying the usual rapid $L$-decrease during the first stages of crystallization is hindered for layer thicknesses approaching the lamellar stack dimensions. Hence, this could suggest that the significant $L$ decrease in the initial stages is associated to processes taking place outside the primary lamellar stacks such as the occurrence of new stacks possibly in the form of lamellar branching, a mechanism described in reference [21].

Fig. 6 also shows that $L$ is significantly larger for the layered materials than for the control sample, especially for the $35 \mathrm{~nm}$ PET thickness layer. Analysis of the last SAXS frame for each isothermal crystallization experiment by means of the Intensity Profile Method [24] following the procedure described in reference [9] yields the $l_{c}$ and $l_{a}$ data collected in Table 2 . It is found that the thickness of the interlamellar amorphous regions tends to increase with decreasing thickness of the PET layer. On the other hand, no trend for $l_{c}$ can be found within the error limits. The results of the present study, including a range of layer thicknesses, are in agreement with preceding published data on a $65 \mathrm{~nm}$ nanolayered PET sample [9] and clearly show that PET lamellar stacks developed under spatial restriction exhibit larger amorphous regions between crystalline lamellae of similar thicknesses than those of the bulk material.

\subsection{Development of crystal orientation as revealed by WAXS}

Fig. 7 illustrates the room temperature WAXS pattern of the $50 \mathrm{~nm}$ layered material, with the incident beam along ND (left) and TD or MD (right). The Bragg spacings associated to the $(h k l)$ reflections are in agreement with the triclinic unit cell early published by Bunn [25]. Isotropic rings are observed with perpendicular incidence while a clear crystal orientation is observed when the beam is directed along any direction within the plane of the layers indicating that the crystal morphology exhibits uniaxial symmetry perpendicularly to the film surface. The pattern resembles the one associated to edge-on lamellar orientation in thin PET nanocomposite films [26] and is similar to the pattern reported for $65 \mathrm{~nm}$ PET layers crystallized at $150^{\circ} \mathrm{C}$ in a multilayer assembly [9]. We have used the CLEARER2 package to simulate uniaxial diffraction patterns of PET with different axis of symmetry allowing for a detailed determination of the crystal orientation [27]. It is shown that the WAXS pattern of Fig. 7b is in agreement with the occurrence of edge-on lamellae, the c-axis randomly oriented in the plane of the layers and the axis of uniaxial symmetry being a few degrees away from the $a-b$ plane [9]. On the other hand, it is noteworthy that the oriented pattern of Fig. 7 could also be explained by means of a model of twisted lamellae, similar to that proposed by Biangardi and Zachmann for low oriented PET [28]. According to this model, lamellae would twist around the $b$-axis, the (001) plane remaining parallel to the surface layers. From the point of view of wide-angle diffraction, this lamellar twisting model is indistinguishable from that in which non-connected isolated crystals are randomly distributed around a uniaxial axis (equivalent to the lamellar twisting axis). However, it should be

Table 2

Average values of the long period, $L$, crystal thickness, $l_{c}$, and amorphous thickness, $l_{a}$, of the lamellar stacks within the different PET layers crystallized at $150{ }^{\circ} \mathrm{C}$. The $l_{a}$ values were determined using the intensity profile method $[9,24]$. The $l_{c}$ values were obtained using: $l_{c}=L-l_{a}$.

\begin{tabular}{lrll}
\hline PET thickness $[\mathrm{nm}]$ & \multicolumn{1}{c}{$L[\mathrm{~nm}]$} & $l_{a}[\mathrm{~nm}]$ & $l_{c}[\mathrm{~nm}]$ \\
\hline Control material & $9.6 \pm 0.1$ & $3.1 \pm 0.3$ & $6.5 \pm 0.4$ \\
115 & $9.9 \pm 0.1$ & $3.2 \pm 0.3$ & $6.7 \pm 0.4$ \\
80 & $10.0 \pm 0.1$ & $3.1 \pm 0.3$ & $6.9 \pm 0.4$ \\
50 & $9.8 \pm 0.1$ & $3.6 \pm 0.3$ & $6.2 \pm 0.4$ \\
35 & $10.8 \pm 0.1$ & $4.7 \pm 0.5$ & $6.1 \pm 0.6$ \\
\hline
\end{tabular}

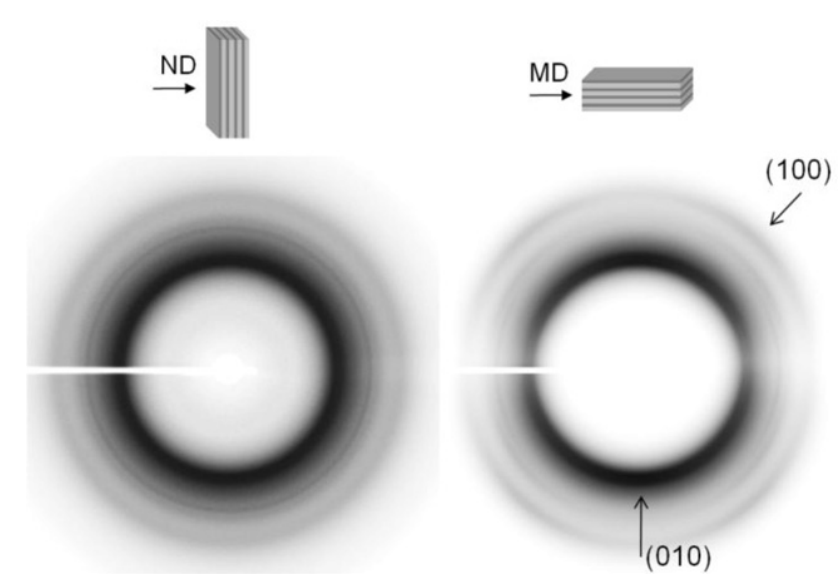

Fig. 7. WAXS patterns of the $50 \mathrm{~nm}$ layered sample after crystallization at $150{ }^{\circ} \mathrm{C}$. The incident beam was directed along the ND (left) and MD (or TD) (right) directions.

noted that the twisting lamellar model should give rise to SAXS maxima on the equator that are absent in our SAXS patterns. In addition, the limited thickness of the PET layers (only a few tenths of nanometres) renders important spatial restrictions to the development of lamellar twisting. Consequently, we envisage, as will be discussed in more detail in the next section, that a lamellar arrangement constituted by rather isolated crystals is more likely to develop.

Most interesting is the observation that the same crystal orientation is preserved at any crystallization temperature and for all the PET layer thicknesses investigated. Indeed, Fig. 8 includes a number of azimuthal scans of the (010) and (100) reflections, for the samples with the thinnest PET layers (50 nm and $35 \mathrm{~nm}$ ) crystallized in the range $117^{\circ} \mathrm{C}-150^{\circ} \mathrm{C}$. In addition, Fig. 9 shows the (010) and (100) azimuthal scans for all the samples after crystallization at $150{ }^{\circ} \mathrm{C}$. It is seen that for the (010) reflection, a maximum of intensity at $90^{\circ}$ and $270^{\circ}$ appears for all the materials and all

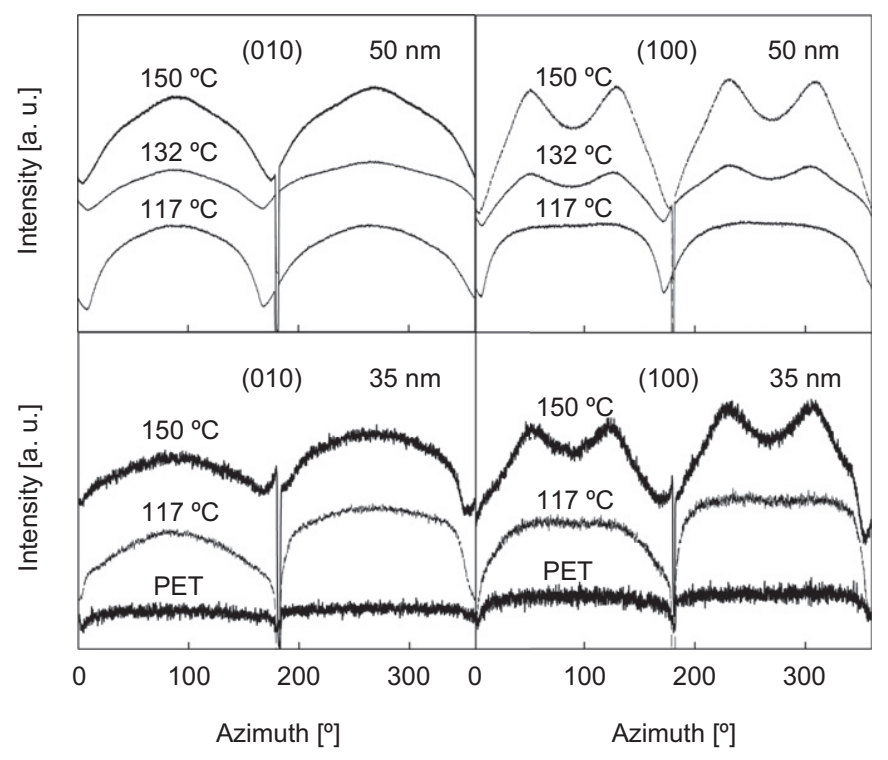

Fig. 8. Diffracted X-ray intensity as a function of azimuthal angle for the $50 \mathrm{~nm}$ and $35 \mathrm{~nm}$ nanolayered PET, crystallized at the indicated temperatures. For each azimuthal angle, the intensity was integrated over a small portion of the $q$ space around the (010) and (100) maxima respectively. Curves are normalized and shifted for the sake of clarity. 


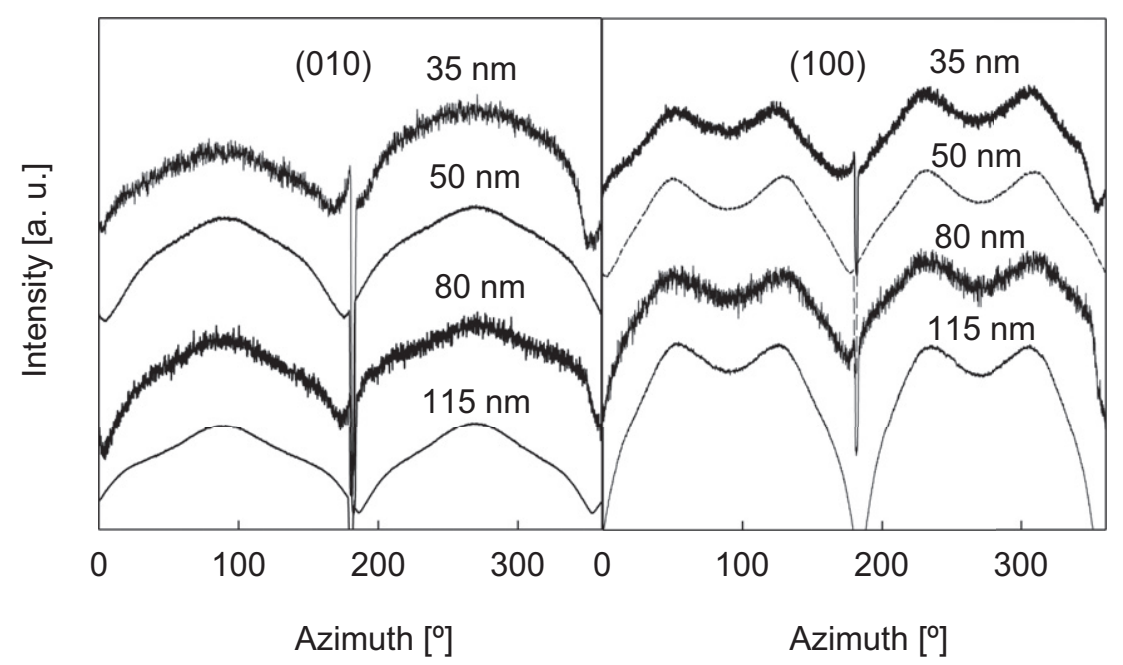

Fig. 9. Intensity trace of the (010) and the (100) reflections as a function of azimuthal angle for the nanolayered series crystallized at $150{ }^{\circ} \mathrm{C}$. Curves are normalized and shifted for the sake of clarity.

crystallization temperatures investigated. In case of the (100) reflection, a double maxima at $\sim 50^{\circ}, 130^{\circ}$ and $\sim 230^{\circ}, 310^{\circ}$ can be distinguished even for the lowest crystallization temperature and the thinnest PET layered material. The conspicuous drop of intensity observed around $0^{\circ}$ and $180^{\circ}$ in all the azimuthal scans should be partly related to the sample absorption. In order to evaluate the relevance of the sample absorption on the azimuthal profiles, the corresponding scans of the control PET sample crystallized at $150{ }^{\circ} \mathrm{C}$ (which should exhibit isotropic rings) has been included in Fig. 8. In contrast to the multilayered samples, the control PET exhibits a clear flat region in the central part of the azimuthal scan.

Fig. 9 reveals that a similar azimuthal profile for both, the (010) and (100) reflections is obtained for all the layer thicknesses investigated. In contrast, a clear broadening of the maxima can be observed as the crystallization temperature decreases (see Fig. 8). The fact that layer thickness does not seem to influence the orientation development suggests that the confinement effects do not account for the appearance of edge-on orientation and this lamellar population most probably arises at the PET/PC interfaces.
3.6. A model for the simultaneous appearance of two lamellar orientations

Results suggest that flat-on and edge-on lamellar populations appear in nanolayered PET. Both lamellar orientations coexist in a wide range of crystallization temperatures $\left(117^{\circ} \mathrm{C}-150{ }^{\circ} \mathrm{C}\right)$ and under severe spatial restrictions (layer thicknesses of $35 \mathrm{~nm}$ ). As shown in preceding sections, flat-on lamellae can be detected by means of SAXS when the incident beam is directed in any direction parallel to the layer stacking. The spatial distribution of flat-on lamellae exhibits uniaxial symmetry along ND. The characteristic anisotropic SAXS patterns (two arcs centred on the meridian) can be observed at all stages of the crystallization process and also upon cooling (results not shown here). The SAXS analysis provided above suggests that flat-on orientation arise as a consequence of spatial confinement. Surprisingly, WAXS studies carried out at room temperature on the crystallized material showed no evidence of maxima associated to flat-on orientation. This result suggests that the crystalline structure of flat-on lamellae is distorted to a great extent possibly due to limitations in the necessary chain mobility to
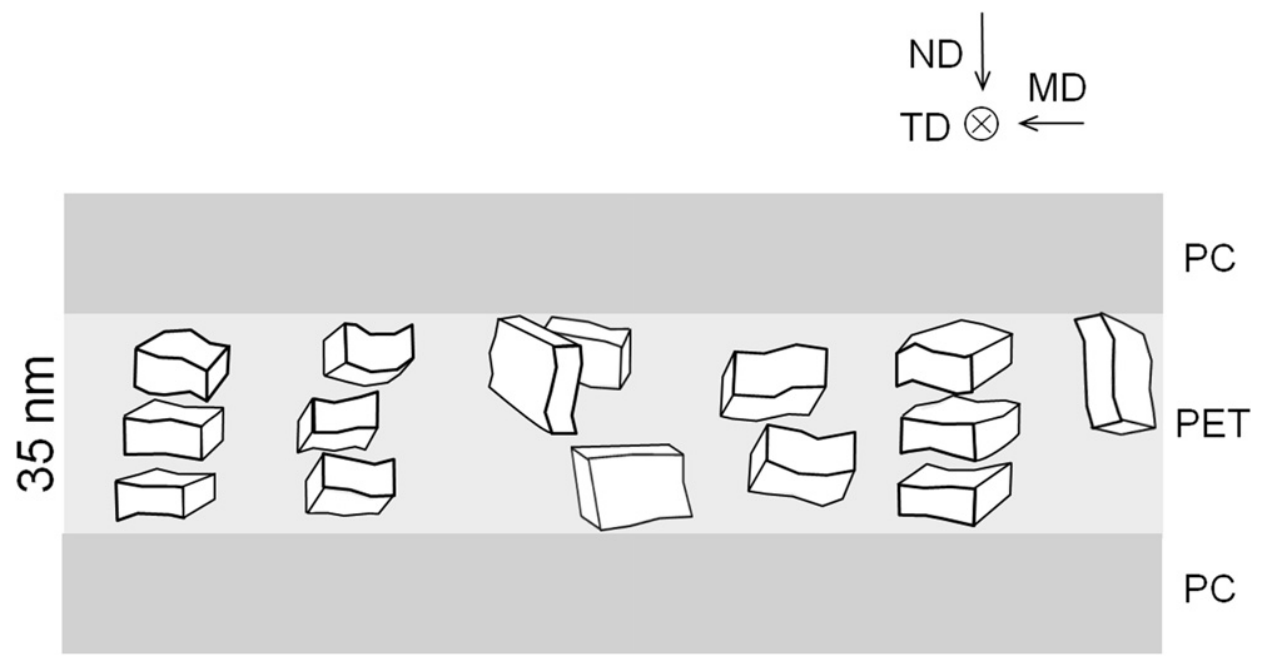

Fig. 10. Schematics of the flat-on and edge-on lamellae arrangement developed within the nanolayered PET (a layer thickness of $35 \mathrm{~nm}$ has been chosen) according to the SAXS and WAXS observations. 
match neighbouring segments; in addition, a limited lateral crystal size could also be expected. These factors should give rise to broad WAXS maxima that would preclude any detection of chain orientation.

On the other hand, room temperature WAXS studies undoubtedly reveal the occurrence of edge-on orientation. The beam incidence along the layer staking clearly show anisotropic scattering. The result suggests a higher perfection and/or a larger lateral size of edge-on crystals with respect to flat-on. The SAXS maximum associated to the edge-on orientation is clearly detected as an isotropic ring when the incident beam is directed perpendicularly to the layer stacking (see references [9] and [11]) due to the uniaxial symmetry of the lamellar crystals. In addition, SAXS analysis suggests that edge-on lamellae develop due to interactions with the interface.

Fig. 10 schematically depicts the most significant features of edge-on and flat-on lamellae. A lamellar arrangement consistent with our X-ray observations has been sketched for the $35 \mathrm{~nm}$ PET layered material. The flat-on lamellar stacks dimensions have been scaled according to the results of Table 2 for the $150{ }^{\circ} \mathrm{C}$ crystallized material. The average number of crystals in a lamellar stack has been represented according to the layer spatial restrictions and to preceding SAXS studies indicating that PET lamellar stacks consist of 3-6 lamellae [9,29].

As a final point, it is worth mentioning that we have not succeeded in finding more direct evidence of the proposed PET lamellar morphology by means of imaging techniques such as AFM and TEM. Obtaining lamellar-scale contrast images in PET seems to be rather difficult as pointed out in a recent paper [30]. Although the multilayered PET/PC nanostructure is easily detected, the obtained images do not offer any clear information on the crystalline structure within PET layers that could confirm the interpretation of our X-ray diffraction results.

\section{Conclusions}

Spatial restrictions are found to promote the appearance of flaton lamellae in nanolayers of PET within an alternated PET/PC stack. In addition, edge-on lamellae seem to appear as a consequence of the interactions with the PC interface. Both lamellar populations coexist in a wide range of cold crystallization temperatures (from $150{ }^{\circ} \mathrm{C}$ up to $117{ }^{\circ} \mathrm{C}$ ) and also in the thinnest layered material investigated $(35 \mathrm{~nm})$. No transition from flat-on to edge-on orientation is observed.

As the thickness of the PET layers is reduced, the population of flat-on lamellae experiences the following changes: i) the orientation distribution narrows, ii) the fraction of oriented crystallized material increases, iii) the crystallization kinetics slows down and iv) the long spacing lamellar periodicity, due to thicker amorphous layers, becomes larger. Results are explained on the basis of spatial restrictions truncating the crystal growth. Narrower distributions of orientation and larger amounts of crystallized material would appear for the thinnest layers due to the fact that lamellar deflection is expected to occur at an earlier stage; for the same reason, kinetics of crystallization should be retarded.

The crystallization temperature influences the distribution of flat-on lamellar orientations. Decreasing the crystallization temperature lowers the orientation parameter $f$, which can be envisaged in terms of enhanced nucleation density and hindered crystal growth for low $T_{c}$. Crystals with smaller lateral dimensions are less liable to experience the influence of spatial limitations during their growth and hence, would develop a broader average distribution of lamellar orientations.

\section{Acknowledgements}

The authors wish to thank the MCI (Ministerio de Ciencia e Innovación), Spain, for funding the research reported under the grant FIS2010-18069. This research was also funded by the NSF Center for Layered Polymeric Systems (grant DMR-0423914). Experiments at HASYLAB (DESY, Hamburg) were generously supported by the German Research Foundation (DFG) and the European Community under contract RII3-CT-2004-506008 (IA-SFS), DESY project II-20070031. The technical support of Dr. J. Perlich at the BW4 beamline (HASYLAB) is greatly appreciated. We are also indebted to the Department of Crystallography (Instituto QuímicaFísica "Rocasolano", CSIC) for providing the facilities to carry out the wide angle X-ray diffraction experiments. We are thankful to Joel Carr (CWRU) for his valuable comments and suggestions.

\section{References}

[1] Wang H, Keum JK, Hiltner A, Baer E, Freeman B, Rozanski A, et al. Science 2009;323:757-60.

[2] Liu YX, Chen EQ. Coord Chem Rev 2010;254:1011-37.

[3] Woo E, Huh J, Jeong YG, Shin K. Phys Rev Lett 2007;98:136103.

[4] Schönherr H, Frank CW. Macromolecules 2003;36:1188-98.

[5] Jin Y, Rogunova M, Hiltner A, Baer E, Nowacki R, Galeski A, et al. J Polym Sci B Polym Phys 2004;42:3380-96.

[6] Wang Y, Chan CM, Ng KM. Macromolecules 2008;41:2548-53.

[7] Ma Y, Hu W, Reiter G. Macromolecules 2006;39:5159-64.

[8] Yang JP, Liao Q Zhou JJ, Jiang X, Wang XH, Zhang Y, et al. Macromolecules 2011;44:3511-6.

[9] Flores A, Arribas C, Fauth F, Khariwala D, Hiltner A, Baer E, et al. Polymer 2010; 51:4530-9.

[10] Ania F, Puente-Orench I, Baltá-Calleja FJ, Khariwala D, Hiltner A, Baer E, et al. Macromol Chem Phys 2008;209:1367-73.

[11] Baltá-Calleja FJ, Ania F, Puente-Orench I, Baer E, Hiltner A, Bernal T, et al. Progr Colloid Polym Sci 2005;130:140-8.

[12] Puente-Orench I, Stribeck N, Ania F, Baer E, Hiltner A, Baltá-Calleja FJ. Polymer 2009;50:2680-7.

[13] Hammersley AP, Svensson SO, Thomson A. Nucl Instrum Meth A 1994;346: $312-21$.

[14] Mitchell GR, Windle AH. Polymer 1983;24:1513-20.

[15] Stribeck N. X-ray scattering of soft matter. Berlin: Springer-Verlag; 2007.

[16] Lu XF, Hay JN. Polymer 2001;42:9423-31.

[17] Wang H, Keum JK, Hiltner A, Baer E. Macromol Rapid Comm 2010;31:356-61.

[18] Wang H, Keum JK, Hiltner A, Baer E. Macromolecules 2010;43:3359-64.

[19] Despotopoulou MM, Frank CW, Miller RD, Rabolt JF. Macromolecules 1996;29: 5797-804.

[20] Jonas AM, Russell TP, Yoon DY. Colloid Polym Sci 1994;272:1344-51.

[21] Wang Z-G, Hsiao BS, Sauer BB, Kampert WG. Polymer 1999;40:4615-27.

[22] Lee B, Shin TJ, Lee SW, Yoon J, Kim J, Youn HS, et al. Polymer 2003;44: 2509-18.

[23] Elsner G, Zachmann HG, Milch JG. Makromol Chem 1981;182:657-63.

[24] Murthy NS, Wang ZG, Hsiao BS. Macromolecules 1999;32:5594-9.

[25] Daubeny RD, Bunn CW, Brown CJ. Proc R Soc A 1954;226:531-42.

[26] Yang H, Bhimaraj P, Yan L, Siegel RW, Schadler LS. J Polym Sci Part B Polym Phys 2007; 45:747-57.

[27] Makin OS, Sikorski P, Serpell LC. J Appl Crystallogr 2007;40:966-72.

[28] Biangardi HJ, Zachmann HG. Makromol Chem 1976;177:1173-84.

[29] Santa Cruz C, Stribeck N, Zachmann HG, Baltá-Calleja FJ. Macromolecules 1991;24:5980-90.

[30] Zia Q Ingolic E, Androsch R. Colloid Polym Sci 2010;288:819-25. 